\title{
Síntomas residuales de la depresión: terapias coadyuvantes
}

\author{
Enrique Octavio Flores Gutiérrez, ${ }^{1}$ Víctor Andrés Terán Camarena, ${ }^{1}$ Jorge Julio González Olvera'
}

Actualización por temas

\section{ABSTRACT}

Residual symptoms of depression (SRD) are those that persist despite a good response to antidepressant drug treatment. They have a high incidence in the psychiatric clinic and are significantly related to a high risk of relapse/recurrence. There are insufficient controlled studies to define a pharmacological treatment for the management of SRD, however, different long-term schemes have proven, nevertheless, that side effects involve a significant limitation. The aim of this review is to investigate and analyze the non-pharmacological treatment options for the management of the SRD. Only four type treatments were found: Cognitive Behavioral Therapy, Well-being Therapy, Mindfulness-Based Cognitive Therapy and Euthymic Therapy. The mentioned psychotherapeutic treatments reported decreased rates in relapse and/or clinimetric decreased in SRD levels.

Key words: Residual symptoms, depression, partial remission, psychotherapy, non-pharmacological treatment, relapse.

\section{INTRODUCCIÓN}

Los Síntomas residuales de la depresión (SRD) son un hecho frecuente en la clínica psiquiátrica, se presentan posterior a la remisión y subsisten a pesar de una farmacoterapia exitosa. Su desatención es un riesgo latente debido a la alta probabilidad de recaída y discapacidad. Los antidepresivos a largo plazo son una solución parcial, ya que los efectos secundarios son razón suficiente para considerar el planteamiento de nuevas estrategias. El objetivo de la presente revisión es conocer y analizar las opciones de tratamiento no farmacológico para el manejo de los SRD. Se han encontrado cuatro tratamientos de tipo psicoterapéutico, los cuales se reportan como una alternativa viable con buenos resultados. La búsqueda de bibliografía se hizo por medio de los buscadores PUBMED, MEDLINE y COCHRANE BVS, utilizando las palabras clave (síntomas residuales, remisión parcial, tratamiento farmacológico, no farmacológico y psicoterapéutico).

\section{RESUMEN}

Los Síntomas Residuales de la Depresión (SRD) son aquellos que persisten a pesar de una buena respuesta al tratamiento farmacológico antidepresivo. Tienen una alta incidencia en la clínica psiquiátrica y se relacionan significativamente con un elevado riesgo de recaída/ recurrencia. No hay suficientes estudios controlados que definan un tratamiento farmacológico en el manejo de SRD, sin embargo, se han probado diferentes esquemas a largo plazo; no obstante, los efectos secundarios implican una gran limitante. El objetivo de la presente revisión es conocer y analizar las opciones de tratamiento no farmacológico para el manejo de los SRD. Sólo se encontraron cuatro tratamientos de tipo psicoterapéutico: la Terapia Cognitivo Conductual, la Terapia del Bienestar, la Terapia Cognitivo Conductual Basada en el Mindfulness y la Terapia Eutímica. Los modelos reportan disminución en las tasas de recaída y/o disminución clinimétrica en los niveles de SRD.

Palabras clave: Síntomas residuales, depresión, remisión parcial, psicoterapia, tratamiento no farmacológico, recaída.

Subdirección de Investigaciones Clínicas. Instituto Nacional de Psiquiatría Ramón de la Fuente Muñiz.

Correspondencia: Doctor Jorge Julio González Olvera. Subdirección de Investigaciones Clínicas. Instituto Nacional de Psiquiatría Ramón de la Fuente Muñiz. Calz. México-Xochimilco 101, San Lorenzo Huipulco, Tlalpan, 14370, México DF., Tel. 4160 - 5349. E-mail: iigonz@gmail.com

Recibido: 13 de enero de 2014. Aceptado: 14 de mayo de 2014. 
caracterizado por el posible aumento de comorbilidades médicas $^{1,2}$ y psiquiátricas, ${ }^{8}$ mayor carga funcional-social ${ }^{9-11}$ e incremento del costo económico. 2,12,13

\section{SÍNTOMAS RESIDUALES DEL TRASTORNO DEPRESIVO}

La naturaleza de los SRD se conforma principalmente por la persistencia de algunos síntomas del TDM, como ánimo bajo, disminución del rendimiento laboral, ansiedad, disfunción sexual, apatía, anergia, sentimientos de culpa, trastornos del sueño, fatiga, reducción de la motivación e irritabilidad. ${ }^{4}$ Del mismo modo, algunos síntomas somáticos, como el dolor de espalda, muscular, abdominal y articular, son también comunes. ${ }^{14}$ La presencia de cada uno de estos síntomas es suficiente para afectar la vida funcional del paciente en su totalidad.

Los SRD son identificados si se alcanza un puntaje $\leq 8$ puntos en la escala del HAM-D-17, o de $\leq 9$ en el Inventario de Depresión de Beck (BDI), ,6,6,15,16 y una puntuación $\leq 10$ en la Escala de Evaluación de la Actividad Global (EEAG). ${ }^{17}$

Los SRD comenzaron a recibir atención antes de los 1990, evidenciados en reportes revisados a detalle, sin embargo su proporción nunca fue bien documentada. ${ }^{18}$ La importancia crece en el momento en que estos se identifican como grandes causantes de discapacidad. ${ }^{18}$ Actualmente, los SRD se relacionan significativamente con un elevado riesgo de recaída tras finalizar un tratamiento antidepresivo; ${ }^{4,5,7,14,15,17-20}$ un fuerte argumento que señala la necesidad de atención prioritaria a este problema.

Los factores considerados de riesgo para la depresión como género, eventos vitales estresantes, experiencias infantiles adversas y ciertos rasgos de personalidad, ${ }^{21}$ pueden jugar un papel causal también para los SRD. Ogrodniczuk et al., en 2004, identificaron que los SRD tienen una asociación significativa con el malestar en general, la disfunción interpersonal y la autoestima. ${ }^{22}$ Algunos autores han mencionado que los SRD pueden estar relacionados con una etiología multifactorial que incluye predisposiciones biológicas, procesos cognitivos vulnerables, estrés ambiental, eventos significativos de la vida y estrés a largo plazo. ${ }^{17}$ En una encuesta entre psiquiatras españoles, realizada por Bousoño et al., en el 2007, las causas que ellos relacionan con los SRD fueron factores de riesgo ambiental y/o de personalidad (40.7\%); tratamiento insuficiente en tiempo y/o dosis (28.5\%); depresión resistente $(25.8 \%)$ y un tratamiento inadecuado (falta de eficacia del antidepresivo elegido, $22.5 \%) .{ }^{4}$

\section{Incidencia de los sintomas residuales}

Varios autores reportan altas tasas de SRD posteriores al tratamiento antidepresivo. En un estudio de 215 pacientes tratados con fluoxetina ( $20 \mathrm{mg} /$ día, por ocho semanas), 108 respondieron al tratamiento $(50.2 \%)$, de los cuales sólo el
$17.6 \%$ no registró SRD, el 25.9\% registró un SRD, y el 56.5\% registró dos o más SRD. ${ }^{23}$ En otra publicación, de los 624 pacientes que respondieron al tratamiento antidepresivo durante tres meses, sólo 412 obtuvieron la remisión, de éstos, el 90\% registró por lo menos un SRD, ${ }^{7}$ porcentaje similar reportado por Nierenberg e Iovieno. ${ }^{24,25}$ Otro estudio con 100 pacientes que recibieron tratamiento farmacológico y/o psicoterapéutico durante tres meses, reportó que el 99\% tenía presencia de SRD, de los cuales el 22\% presentaba cuatro síntomas. ${ }^{20}$ En un último trabajo a mencionar, de 108 pacientes con TDM evaluados y tratados por nueve meses, 79 (73.1\%) fueron considerados remitentes, sin embargo, de ellos, el $82.3 \%$ presentaron SRD. ${ }^{26}$ El cuadro 1 muestra otros datos que confirman el alcance de los SRD. La alta incidencia de SRD en la clínica ratifica la consideración de abordar nuevas investigaciones sobre su manejo.

\section{Dimensiones específicas en los síntomas residuales}

La importancia que tiene el abordaje de los dominios específicos, es decir, las características nosológicas de los SRD, es conocer si afectan en mayor o menor medida al desempeño funcional del paciente, si algunos se asocian más a la recurrencia y recaída, o si requieren un tratamiento dominio específico.

Según Romera et al., pocos trabajos han evaluado el impacto de SRD sobre el deterioro funcional. En su publicación reportó que la asociación entre los dominios de síntomas residuales (estado de ánimo, insomnio, ansiedad, síntomas somáticos y síntomas de dolor) y el funcionamiento del paciente, difiere según el tipo de síntomas. ${ }^{7}$ Además, afirma de forma preliminar que algunos de los SRD presentan un mayor riesgo de recaída que otros.

Por otra parte, Karp et al. sugieren que independientemente del tipo de tratamiento, el alto nivel de variabilidad de los SRD está asociado a un elevado riesgo de recurrencia. ${ }^{17}$ En línea con esto, varios estudios recientes han tratado de identificar cuáles SRD son más frecuentes y aquellos que pueden ser predictivos de recaída o recurrencia, encontrando a la ansiedad como uno de los más recurrentes. ${ }^{7,15,27-31}$

En algunas de las publicaciones revisadas, se habla de la presencia o ausencia de SRD como grupos de síntomas y no se describe el dominio específico de cada uno. Menza et al. advierten que la identificación de patrones específicos de SRD individuales puede ser una guía para las opciones de tratamiento y para propiciar mejores resultados a largo plazo. ${ }^{19}$ De este modo, el abordaje de los SRD con tratamientos específicos, es una sugerencia importante a considerar en lo que se refiere a la planeación de nuevas estrategias para su manejo.

\section{Tratamiento farmacológio de síntomas residuales}

Debido a la innegable relación que existe entre los SRD y la recaída o recurrencia, se han propuesto diferentes esquemas de tratamiento en la fase de mantenimiento, enfocados en la prevención de dichos eventos. Un método ampliamente 


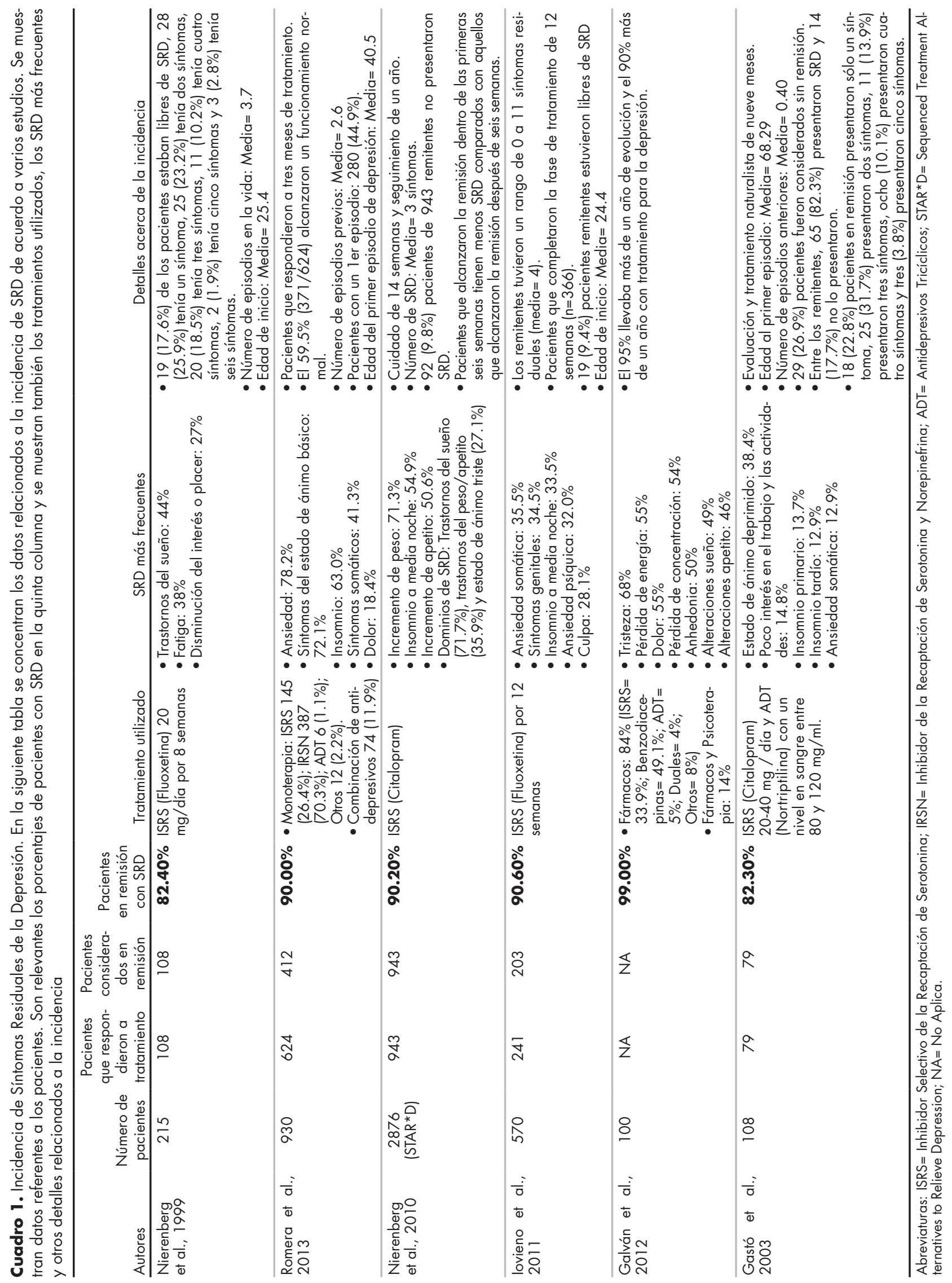


aceptado es la farmacoterapia a largo plazo, para ello, se sugieren dosis completas de antidepresivos durante al menos un año, aunque el límite superior no está bien definido. ${ }^{32}$ Sin embargo, debido a que el tratamiento de mantenimiento antidepresivo se aplica de acuerdo a los lineamientos de las Guías Clínicas para el Tratamiento de Trastornos Psiquiátri$\cos (\mathrm{APA}),{ }^{33}$ es esencial que sólo los pacientes que están en alto riesgo de recurrencia depresiva sean considerados para un tratamiento a largo plazo. ${ }^{32}$ El riesgo de efectos secundarios en comparación con el riesgo de recurrencia puede obligar a la interrupción del tratamiento, con la opción de reiniciarlo a la primera señal de una depresión recurrente. ${ }^{32}$

Por ahora, hay insuficientes estudios controlados de farmacoterapia que sean capaces de proporcionar algún tratamiento claro para tratar específicamente los SRD. ${ }^{14}$ Sin embargo, actualmente, las estrategias farmacológicas primarias disponibles para tratar los SRD son: aumentar el tiempo con la misma medicación, un cambio de medicamento, el uso de tratamientos secuenciales (primero medicamento, seguido de psicoterapia) y el aumento o combinación de tratamientos adicionales. Esta última es considerada como una estrategia de primera línea, ya que los pacientes que, por el contrario, son tratados con monoterapia (por ejemplo, fluoxetina, citalopram o reboxetina) continúan experimentando $\mathrm{SRD}^{23-25,30}$ y tienen bajas tasas de remisión completa. ${ }^{14}$ Algunos antidepresivos utilizados en la prevención de la recaída o recurrencia del TDM son: la imipramina, la fluoxetina, la sertralina, la paroxetina, el citalopram, la venlafaxina. ${ }^{32}$ La duloxetina, especialmente indicada en depresión con componente doloroso, es sugerida ante SRD. ${ }^{34}$ Mientras que la buspirona, el modafinilo y el folato son otros tratamientos con alguna posibilidad de beneficio en el tratamiento de los SRD. ${ }^{14}$ Ante todo esto, algunas investigaciones sugieren que la psicoterapia tiene un papel importante en optimizar los efectos del tratamiento farmacológico y en mejorar el pronóstico de los pacientes a largo plazo, demostrando ser eficaz en la prevención de episodios adicionales de la depresión. . $2,35^{2}$

\section{TRATAMIENTO NO FARMACOLÓGICO DE LOS SÍNTOMAS RESIDUALES}

Las implicaciones más importantes de los SRD son los siguientes: el pronóstico de discapacidad, la recaída o recurrencia y la necesidad de tratamiento (dirigido a los SRD y/o profiláctico para prevenir la recaída). ${ }^{18}$ Es fundamental evaluar en primera instancia si los SRD son parte del TDM, si son un efecto secundario del tratamiento antidepresivo o una comorbilidad. ${ }^{19}$ Asimismo, considerar el inicio de una adecuada psicoeducación, el establecimiento de una adecuada dosis y una apropiada duración del tratamiento farmacológico. ${ }^{14}$

Pese al éxito del tratamiento farmacológico antidepresivo en fase aguda, los pacientes con SRD presentan un ma- yor riesgo de recaída o recurrencia, en comparación con los pacientes sin $\mathrm{SRD},{ }^{35}$ por lo que es importante establecer un tratamiento de mantenimiento adecuado según el perfil del paciente. La psicoterapia es un recurso auxiliar muy efectivo ante esta problemática.

De acuerdo con la Asociación Psiquiátrica Americana (APA), el uso de psicoterapia sola, centrada en la depresión, se recomienda como una opción de tratamiento inicial para pacientes con TDM de leve a moderada intensidad. ${ }^{36}$ Mientras que la combinación de psicoterapia y psicofármacos puede ser usada como un tratamiento inicial para pacientes con TDM de moderada a grave intensidad. ${ }^{36}$ Los factores que sugieren el uso de intervenciones psicoterapéuticas son: presencia de estrés psicosocial significativo, conflicto intrapsíquico, dificultades interpersonales, un trastorno en comorbilidad del Eje II, la disponibilidad de tratamiento y/o, sobre todo, la preferencia del paciente. ${ }^{36}$

El uso de psicoterapia como adyuvante al tratamiento farmacológico se puede aplicar de forma simultánea o secuencial. ${ }^{35}$ En una revisión realizada por Petersen, se analizaron trabajos de intervención psicoterapéutica de forma simultánea (en fase aguda o de mantenimiento) y de forma secuencial (enfoque que aborda la presencia de SRD). ${ }^{35} \mathrm{El}$ autor concluye que la aplicación simultánea de la farmacoterapia y psicoterapia durante la fase aguda del tratamiento sólo parece proporcionar un modesto aumento en las tasas de respuesta, aunque puede prevenir o retrasar la recaída, mientras que en la fase de mantenimiento no proporciona ventaja sobre el mantenimiento de la farmacoterapia. Por el contrario, el uso secuencial de la psicoterapia después de la remisión con tratamiento médico antidepresivo agudo, confiere un mejor pronóstico a largo plazo, en términos de prevención de recaídas o recurrencias (en comparación con una intervención simultánea). Esta puede ser una alternativa viable como tratamiento de mantenimiento con medicamentos para algunos pacientes. ${ }^{35}$

Los posibles mecanismos de mejora por la psicoterapia como adyuvante en el tratamiento del TDM, de acuerdo con Petersen, son: ${ }^{35} 1$. Incrementa la reducción de síntomas y promueve la mejoría funcional (aumenta tasas de remisión y reduce tasas de recaída o recurrencia). 2. Mejora los SRD que persisten después del tratamiento antidepresivo agudo, previniendo su progresión hacia síntomas prodrómicos de recaída. 3. Se enfoca o dirige a síntomas específicos asociados con la recaída (culpa, desesperanza, pesimismo, baja autoestima) mejor que los antidepresivos. 4. Aumenta las habilidades de afrontamiento, importantes para el manejo a largo plazo de la enfermedad. 5. Promueve el mantenimiento de los cambios saludables en las construcciones cognitivas asociadas con la respuesta aguda y remisión, durante el tratamiento de continuación y mantenimiento. 6. La psicoterapia se dirige a zonas cerebrales distintas que los antidepresivos, datos de neuroimagen sugieren efectos diferenciales y moduladores beneficiosos sobre el sistema córtico-límbico. ${ }^{35}$ 
Existen una serie de publicaciones que demuestran la eficacia del uso de psicoterapia en el manejo de SRD, estos son: la Terapia Cognitivo Conductual, la Terapia del Bienestar, la Terapia Cognitivo Conductual Basada en el Mindfulness y la Terapia Eutímica.

\section{Terapia cognitivo conductual}

El uso de la terapia cognitivo conductual (TCC) mostró eficacia en pacientes con SRD en dos estudios. En ambos se encontró que la adición de TCC al régimen farmacológico, que anteriormente había dado respuesta parcial, generó una reducción en las tasas de recaída. En el primero, Fava et al. reportaron una diferencia en las tasas de recaída a los cuatro años de seguimiento en un grupo de 40 pacientes; el grupo control con exclusivo manejo clínico tuvo tasas de recaída del 70\%, en tanto que el grupo con TCC reportó tasas de recaída del 35\%, en ambos grupos los antidepresivos fueron reducidos y descontinuados. ${ }^{37} \mathrm{El}$ otro estudio, de Paykel et al., presenta importantes diferencias respecto al anterior, ya que trabajaron con criterios más rigurosos de investigación, mayores niveles clinimétricos de SRD, una TCC modificada y un número de pacientes mayor (158), encontrando que la adición de TCC a la medicación reduce las tasas de recaída en 68 semanas, demostrado por un 47\% del grupo con únicamente medicación y un $29 \%$ del grupo con TCC; ambos recibieron continuación y mantenimiento de antidepresivos a la misma dosis durante todo el trabajo. ${ }^{16}$ Sin embargo, a pesar de comprobarse una menor tasa de recaída con el uso de TCC, no existió disminución significativa de los SRD posiblemente, y a falta de ser verificado, se origina por el mecanismo compensatorio de la TCC, que enseña al paciente cómo hacer frente a los síntomas persistentes, influyendo, por tanto, únicamente en la evitación de recaída, pero no en la disminución como tal de los niveles de SRD. ${ }^{38}$ En relación con este efecto, Perlis et al. reportan que el aumento de dosis de fluoxetina (40mg) en combinación con TCC durante la fase de continuación en pacientes con SRD, no representó un beneficio significativo sobre las tasas de recaída ni en la disminución de SRD en comparación con el uso de farmacoterapia sola. ${ }^{39}$ Es preciso realizar nuevas investigaciones que aclaren este efecto.

En los últimos años han surgido nuevas modificaciones a la TCC, las cuales han sido experimentadas en el tratamiento de SRD, después de la descontinuación de los antidepresivos. Entre éstas, se han propuesto a la Terapia del Bienestar (TB) (WBT, por sus siglas en inglés) y el tratamiento con TCC basado en el Mindfulness (MBCT, por sus siglas en inglés).

\section{TCC-terapia del bienestar}

La TB basada en el modelo cognitivo de Ryff, que utiliza técnicas que enfatizan la auto-observación, el uso de un diario estructurado y la interacción entre los pacientes y los terapeutas, ${ }^{40}$ fue probada en un estudio de 40 pacientes con TDM recurrente, tratados exitosamente con antidepresivos.
Fueron asignados al azar a un grupo con TB y farmacoterapia o a un grupo con farmacoterapia y gestión clínica; en ambos grupos se redujeron y descontinuaron los antidepresivos durante el experimento. En sus resultados a dos años de seguimiento, la TB dio muestra de una tasa de recaída más baja (25\%) que la gestión clínica $(80 \%) .{ }^{40}$

\section{Terapia cognitivo conductual basada en el Mindfulness}

El MBCT ha sido una intervención eficaz. ${ }^{41}$ Un estudio diseñado para enseñar a pacientes recurrentes del TDM a desvincularse de la disforia y de sus rutinas cognitivas disfuncionales (rumiación depresiva), conformó 145 pacientes, asignados aleatoriamente en alguno de estos dos grupos: tratamiento usual o tratamiento usual sumado a MBCT. Durante 60 semanas de seguimiento, los pacientes que se encontraban en alto riesgo de recaída (con tres o más episodios previos) y habían recibido $\mathrm{MBCT}$ tuvieron una probabilidad del 37\% de deprimirse nuevamente, mientras que el grupo que recibió sólo tratamiento usual tuvo una probabilidad de recaer del $66 \%$. Sin embargo, en aquellos pacientes con sólo dos episodios depresivos previos no se mostró evidencia de beneficio. ${ }^{42} \mathrm{Un}$ resultado que demuestra que esta intervención puede reducir significativamente el riesgo de recaída y recurrencia en pacientes que han experimentado tres o más episodios previos del TDM. Estos resultados se confirmaron con la replicación del mismo estudio más adelante. ${ }^{43}$ Otra intervención el MBCT también mostró resultados prometedores al disminuir significativamente la rumiación excesiva en pacientes con SRD. ${ }^{44}$

\section{Terapia eutímica}

La terapia eutímica (TE) fue evaluada y comparada en la reducción de SRD con un grupo activo de Psicoeducación (PE) por Kiermeir et al. ${ }^{45}$ El trabajo incluyó a 46 pacientes ambulatorios, con remisión parcial del TDM, asignados aleatoriamente en un grupo de TE $(n=23)$ o en un grupo de PE $(n=23)$. En ambos se continuó el tratamiento usual de medicamentos y se les realizó un seguimiento de tres meses. Los resultados muestran que la TE reduce los SRD tan eficazmente como la $\mathrm{PE}$, dado que ambos grupos mostraron una disminución significativa en la severidad de la depresión (medida por el BDI-II y en los SRD, medida por el HAM-D-21 después de la intervención), manteniéndose estable en los tres meses de seguimiento, durante el cual no se administró un tratamiento adicional. Por otra parte, después de la intervención se observó una tendencia al aumento en el autocuidado, medido por el Marburg self-care questionaire (MR FSF, por sus siglas en inglés). El estudio sugiere que la ET puede fortalecer la adherencia al tratamiento en el paciente. ${ }^{45}$

Los cuatro modelos psicoterapéuticos, pese a sus diferencias metodológicas, presentan resultados interesantes, lo cual habla de la importancia que desempeña la psicoterapia como tratamiento auxiliar de los SRD. El cuadro 2 resume los objetivos terapéuticos, las tasas de recaída, los resultados clinimétricos y las conclusiones más importantes de esos estudios. 


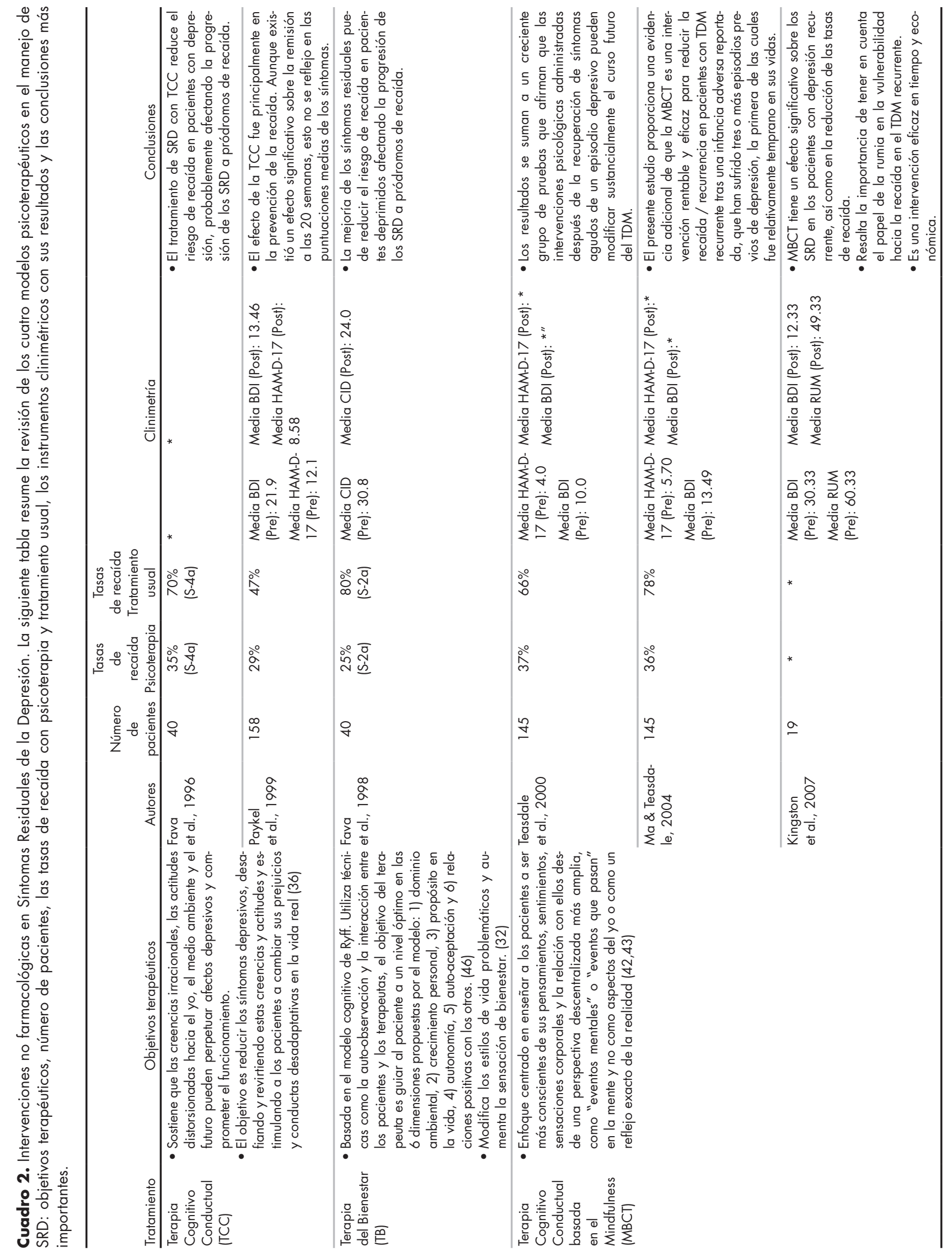




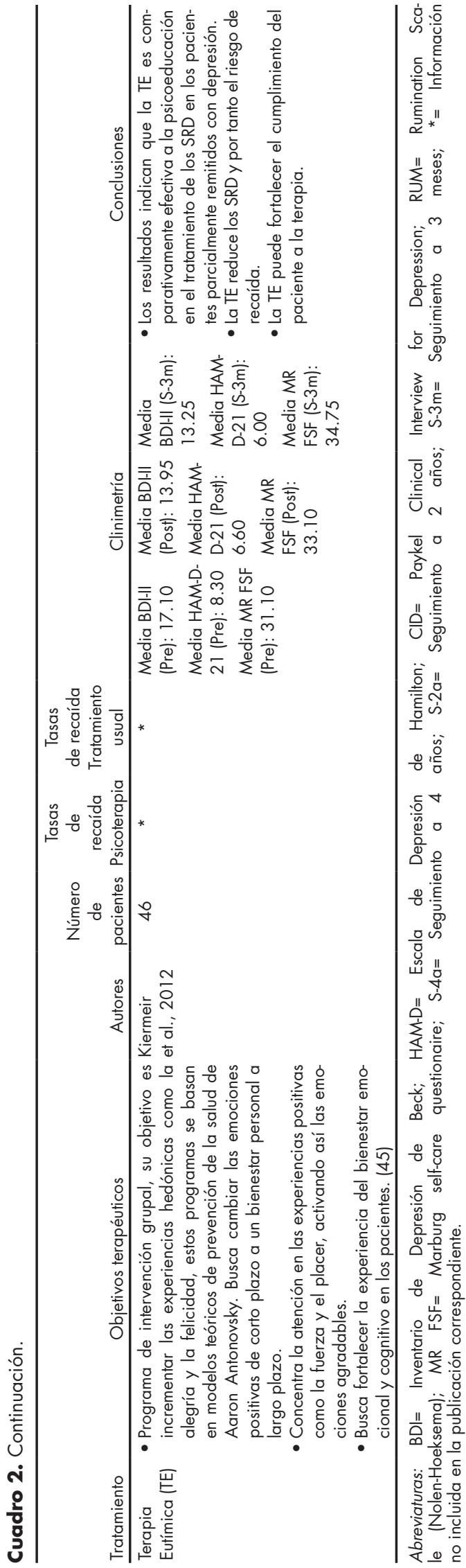

DISCUSIÓN

Hemos presentado datos relevantes relacionados con la incidencia, prevalencia y características de los SRD en la clínica (cuadro 1), que alertan sobre la necesidad de buscar nuevas y diferentes opciones de tratamiento. De ahí la importancia que tuvo el realizar una revisión del tratamiento no farmacológico de SRD, en la cual sólo encontramos algunos de tipo psicoterapéutico enfocados en su manejo. De esta búsqueda, los datos que encontramos más destacados son los siguientes:

\section{Importancia de los SRD}

Los SRD son frecuentes en la clínica, se asocian con recaída, recurrencia y discapacidad. Comprobadas por gran cantidad de investigaciones, ${ }^{4,5,7,9-11,14,15,17-20}$ se sugiere la necesidad de un abordaje específico ${ }^{19}$ enfocado en estrategias para el manejo de SRD. La etiología de SRD es aún imprecisa, por lo que se recomienda al clínico realizar una evaluación exhaustiva ${ }^{14}$ para identificar si la presencia de SRD está relacionada a efectos secundarios del tratamiento antidepresivo, comorbilidad, antecedentes adversos de la infancia, ${ }^{43}$ o síntomas persistentes del propio trastorno depresivo. ${ }^{19}$ Ante esta problemática son necesarias investigaciones para establecer criterios etiológicos y diagnósticos más claros, los cuales permitirán una mejor definición operativa de los SRD. Este paso favorecería la creación de algoritmos clínicos de tratamiento, estrategias más eficientes y mayores beneficios a largo plazo.

\section{Repercusión del tratamiento farmacológico}

La íntima relación entre los SRD y las altas tasas de recaí$\mathrm{da} /$ recurrencia han llevado a proponer diferentes esquemas de tratamiento farmacológico a largo plazo dirigidos a evitar esas consecuencias. En varios estudios, a pesar del uso de monoterapia o de la combinación de psicofármacos, ${ }^{7,20,23-26}$ se mantienen altas tasas de incidencia de SRD. Los psicofármacos pueden ocasionar una gran variedad de efectos secundarios que perturban al paciente, provocando el abandono del tratamiento $\mathrm{y}$, eventualmente, la recurrencia del trastorno depresivo..$^{32}$ En este punto, la psicoterapia puede tener un papel importante en la adherencia al tratamiento y en la optimización de la respuesta a los antidepresivos, logrando eficacia en la prevención de nuevos episodios. ${ }^{32,35}$ En línea con esto, Petersen (2006) concluye que, en la prevención de recaídas, el uso secuencial de psicoterapia (después de alcanzar la remisión con la farmacoterapia antidepresiva) puede otorgar un mejor pronóstico a largo plazo que su uso simultáneo. ${ }^{35}$

\section{Ventajas del abordaje psicoterapéutico}

El uso de psicoterapia como coadyuvante en el tratamiento de los SRD se puede enfocar en superar dificultades o disfunciones asociadas a factores psicosociales característicos 
de este padecimiento, tales como disfunción en las relaciones interpersonales, estrés ambiental a largo plazo, eventos significativos de la vida, procesos cognitivos vulnerables, problemas de personalidad, baja autoestima y malestar en general, entre otros. ${ }^{4,17,22,25}$ Esto, por medio de estrategias orientadas a la reducción de la magnitud de síntomas específicos (como culpa, desesperanza, pesimismo, baja autoestima), para promover mejoría funcional, habilidades de afrontamiento, construcciones cognitivas más racionales, entre otras. Todo lo cual favorece las tasas de remisión y reduce las tasas de recaída/recurrencia. . $^{32,35,36}$

\section{Modelos psicoterapéuticos en el manejo de SRD}

Los cuatro modelos psicoterapéuticos utilizados como opción en el manejo de SRD son: Terapia cognitivo conductual, ${ }^{16,37}$ Terapia del bienestar, ${ }^{40}$ Terapia cognitivo conductual basada en el Mindfulness ${ }^{42-44}$ y Terapia eutímica. ${ }^{45}$ Todos fueron aplicados tras la remisión por tratamiento farmacológico y ante la persistencia de SRD. Los cuatro modelos comparten objetivos terapéuticos esenciales, tales como propiciar un equilibrio cognitivo, eutímico y el reconocimiento de conductas o estilos de vida disfuncionales. Sin embargo, las diferencias radican en su enfoque: la TCC y la MBCT buscan destacar la implicación de los procesos mentales distorsionados (rumiación), mientras que la TB y la TE se centran en incrementar la experiencia del bienestar por medio de experiencias positivas hedónicas que activen emociones agradables. Asimismo, tanto la TCC como la MBCT se caracterizan por propiciar un enfoque hacia la auto-observación, mientras que la TB y la TE destacan por un enfoque que propicia la interacción con el otro. A pesar de la similitud existente entre la TCC y la MBCT, en cuanto a destacar la implicación de la rumiación, existen diferencias destacadas en su manejo, dado que la TCC busca la modificación y el desafío de creencias y procesos mentales, ${ }^{36}$ mientras que la MBCT no busca modificar ni desafiar, sino propiciar una perspectiva descentralizada que considere a los procesos rumiantes como "eventos mentales que pasan" y que no forman parte de una representación aproximada de la realidad. ${ }^{42,43}$

El uso de psicoterapia simultánea enfocada específicamente en SRD, reporta bajas tasas de recaída en comparación con el tratamiento sin psicoterapia, tanto en la TCC, ${ }^{16,37}$ en $\mathrm{TB}_{,}{ }^{40}$ como en MBCT. ${ }^{42,43}$ En el uso de psicoterapia secuencial, en los casos de $\mathrm{MBCT}^{44}$ y TE, ${ }^{45}$ las tasas de recaída no fueron reportadas, aunque sí se reporta disminución en los niveles de SRD medidos con clinimetría.

\section{CONCLUSIÓN}

Finalmente, la importancia tanto de esta revisión como de los resultados reportados radica en su futura consideración para las nuevas propuestas terapéuticas, junto a la posibilidad de perfilar manejos aún más precisos que beneficien a largo plazo al paciente con SRD, y que al mismo tiempo se conviertan en líneas de investigación para desarrollar nuevas alternativas de tratamiento confiables, efectivas y replicables.

\section{Financiamiento}

No aplica.

\section{Declaración de conflicto de intereses}

Los autores declararon no tener conflicto de intereses.

\section{REFERENCIAS}

1. Wagner FA, González-Forteza C, Sánchez-García S, García-Peña C et al. Enfocando la depresión como problema de salud pública en México. Salud Mental 2012;35(1):3-11.

2. McIntyre RS, $\mathrm{O}^{\prime}$ Donovan $\mathrm{C}$. The human cost of not achieving full remission in depression. Can J Psychiatry 2004;49(1):10s-16s.

3. Casquero RR, Benítez MJM, Manzanera ER, Mínguez VJC et al. Guía de buena práctica clínica en depresión y ansiedad generalizada con presentación de síntomas somáticos. Madrid: Ed. Organización Médica Colegial de España; 2012.

4. Bousoño GM, Camacho LM, Pérez PJF. Síntomas residuales y remisión en la depresión: una encuesta de opinión entre psiquiatras españoles. Psiquiatr Biol 2007;14(3):85-91.

5. Tranter R, O’Donovan C, Chandarana P, Kennedy S. Prevalence and outcome of partial remission in depression. J Psychiatry Neurosci 2002;27(4):241-247.

6. Zajecka JM. Treating depression to remission. J Clin Psychiatry 2003;64(15):7-12.

7. Romera I, Pérez V, Ciudad A, Caballero L et al. Residual symptoms and functioning in depression, does the type of residual symptom matter? A post-hoc analysis. BMC Psychiatry 2013;13(51):1-7. doi:10.1186/1471244X-13-51.

8. Enns MW, Swenson JR, McIntyre RS, Swinson RP et al. Clinical guidelines for the treatment of depressive disorders VII comorbidity. Can J Psychiatry 2001;46(1):77s-90s.

9. Goldberg RJ, Steury S. Depression in the Workplace: Costs and Barriers to treatment. Psychiatr Serv 2001;52(12):1639-1643. doi:10.1176/ appi.ps.52.12.1639.

10. Kessler RC, Barber C, Birnbaum HG, Frank RG et al. Depression in the workplace: Effects on short-term disability. Health Aff 1999;18(5):163171. doi:10.1377/hlthaff.18.5.163.

11. Druss BG, Schlesinger M, Allen HM. Depressive symptoms, satisfaction with health care, and 2-year work outcomes in an employed population. Am J Psychiatry 2001;158:731-734.

12. Lara-Muñoz MC, Robles-García R, Orozco R, Real $T$ et al. Estudio de costo-efectividad del tratamiento de la depresión en México. Salud Mental 2010;33(4):301-308.

13. Sobocki P, Ekman M, Agren H, Runeson B et al. The mission is remission: health economic consequences of achieving full remission with antidepressant treatment for depression. Int J Clin Pract 2006;60(7):791-798.

14. Israel JA. The impact of residual symptoms in major depression. Pharmaceuticals 2010;3:2426-2440. ISSN 1424-8247. doi:10.3390/ph3082426.

15. Taylor DJ, Walters HM, Vittengl JR, Krebaum S et al. Which depressive symptoms remain after response to cognitive therapy of depression and predict relapse and recurrence? J Affect Disord 2010;123(1-3):181187. doi:10.1016/j.jad.2009.08.007.

16. Paykel ES, Scott J, Teasdale JD, Johnson AL et al. Prevention of relapse in residual depression by cognitive therapy. Arch Gen Psychiatry 1999;56:829-835. 
17. Karp JF, Buysse, DJ, Houck PR, Cherry C et al. Relationship of variability in residual symptoms with recurrence of major depressive disorder during maintenance treatment. Am J Psychiatry 2004;161:1877-1884.

18. Paykel ES. Residual symptoms and relapse in depression. Medicographia 2009;31(2):157-163.

19. Menza M, Marin H, Sokol OR. Residual symptoms in depression: Can treatment be symptom-specific? J Clin Psychiatry 2003;64(5):516-523.

20. Galván P, Fernández E, Fonseca J. Síntomas residuales en el anciano con depresión. Semergen 2012;38(5):285-291. doi:10.1016/ j.semerg.2011.08.006.

21. Silva H. Nuevas perspectivas en la biología de la depresión. Rev chil neuro-psiquiatr [online] 2002;40(1):9-20. ISSN 0717-9227. doi:10.4067/ S0717-92272002000500002.

22. Ogrodniczuk JS, Piper WE, Joyce AS. Residual symptoms in depressed patients who successfully respond to short-term psychotherapy. J Affect Disord 2004;82(3):469-473.

23. Nierenberg AA, Keefe BR, Leslie VC, Alpert JE et al. Residual symptoms in depressed patients who respond acutely to fluoxetine. J Clin Psychiatry 1999;60(4):221-225.

24. Nierenberg AA, Husain MM, Trivedi MH, Fava $M$ et al. Residual symptoms after remission of major depressive disorder with citalopram and risk of relapse: a STAR*D report. Psychol Med 2010;40(1):4150. doi:10.1017/S0033291709006011.

25. Iovieno N, Van Nieuwenhuizen A, Clain A, Baer L et al. Residual symptoms after remission of major depressive disorder with fluoxetina and risk of relapse. Depress Anxiety 2011;28(2):137-144. doi:10.1002/ da.20768.

26. Gastó C, Navarro V, Catalán R, Portella MJ et al. Residual symptoms in elderly major depression remitters. Acta Psychiatr Scand 2003;108(1):15-19. doi:10.1034/j.1600-0447.2003.00068.x.

27. Dombrovski AY, Mulsant BH, Houck PR, Mazumdar S, Lenze EJ, Andreescu C, Cyranowski JM, Reynolds CF. Residual symptoms and recurrence during maintenance treatment of late-life depression. J Affect Disord 2007;103(1-3):77-82. doi:10.1016/j.jad.2007.01.020.

28. Vieta E, Sánchez-Moreno J, Lahuerta J, Zaragoza S. Subsyndromal depressive symptoms in patients with bipolar and unipolar disorder during clinical remission. J Affect Disord 2008;107(1-3):169-174.

29. Yang H, Chuzi S, Sinicropi-Yao L, Johnson D et al. Type of residual symptom and risk of relapse during the continuation/maintenance phase treatment of major depressive disorder with the selective serotonin reuptake inhibitor fluoxetine. Eur Arch Psychiatry Clin Neurosci 2010;260(2):145-150. doi:10.1007/s00406-009-0031-3.

30. Nelson JC, Portera L, Leon AC. Residual symptoms in depressed patients after treatment with fluoxetine or reboxetine. J Clin Psychiatry 2005;66(11):1409-1414.

31. McIntyre RS, Konarski JZ, Soczynska JK, Kennedy SH. Residual anxiety symptoms in depressed primary care patients. J Psychiatr Pract 2007;13(2):125-128.
32. Nierenberg AA, Petersen TJ, Alpert JE. Prevention of relapse and recurrence in depression: The role of long-term pharmacotherapy and psychotherapy. J Clin Psychiatry 2003;64(15):13-17.

33. Karasu TB, Gelenberg A, Merriam A, Wang P. Guía clínica para el tratamiento del trastorno depresivo mayor. Segunda edición. En: American Psychiatric Association (ed). Guías clínicas para el tratamiento de los trastornos psiquiátricos: Compendio 2006. Barcelona: Ars Medica; 2006.

34. Madueño CAJ, Arbesú PJA, Gonçalves EF, Pérez SV et al. Guía de buena práctica clínica en algoritmos de decisión en depresión. Madrid: Ed. Organización Médica Colegial de España; 2011.

35. Petersen TJ. Enhancing the efficacy of antidepressants with psychotherapy. J Psychopharmacol 2006;20(3):19-28.

36. American Psychiatric Association. Practice guideline for the treatment of patients with major depressive disorder. 3rd Ed. Washington: American Psychiatric Association Publishing Inc; 2010.

37. Fava GA, Grandi S, Zielezny M, Rafanelli C et al. Four-year outcome for cognitive behavioral treatment of residual symptoms in major depression. Am J Psychiatry 1996;153(7):945-947.

38. Scott J, Teasdale JD, Paykel ES, Johnson AL et al. Effects of cognitive therapy on psychological symptoms and social functioning in residual depression. Br J Psychiatry 2000;177:440-446.

39. Perlis RH, Nierenberg AA, Alpert JE, Pava J et al. Effects of adding cognitive therapy to fluoxetine dose increase on risk of relapse and residual depressive symptoms in continuation treatment of major depressive disorder. J Clin Psychopharmacol 2002;22(5):474-480.

40. Fava GA, Rafanelli C, Grandi S, Conti S et al. Prevention of recurrent depression with cognitive behavioral therapy: preliminary findings. Arch Gen Psychiatry 1998;55(9):816-820.

41. Piet J, Hougaard E. The effect of mindfulness-based cognitive therapy for prevention of relapse in recurrent major depressive disorder: A systematic review and meta-analysis. Clin Psychol Rev 2011;31(6):1032-1040.

42. Teasdale JD, Segal ZV, Williams JMG, Ridgeway VA et al. Prevention of relapse/recurrence in major depression by mindfulness-based cognitive therapy. J Consult Clin Psychol 2000;68(4):615-623.

43. Ma SH, Teasdale JD. Mindfulness-based cognitive therapy for depression: Replication and exploration of differential relapse prevention effects. J Consult Clin Psychol 2004;72(1):31-40. doi:10.1037/0022006X.72.1.31.

44. Kingston T, Dooley B, Bates A, Lawlor E et al. Mindfulness-based cognitive therapy for residual depressive symptoms. Psychol Psychother 2007;80(2):193-203. doi:10.1348/147608306X116016.

45. Kiermeir J, Gassner LM, Siebörger A, Wiethoff K et al. Euthymic therapy to reduce residual symptoms of depression and strengthen self-care a randomised controlled trial. German J Psychiatry 2012;15(1):15-22. 